\title{
Complete Polarization Control for a Nanofiber Waveguide Using Directional Coupling
}

\author{
Fuchuan Lei, Georgiy Tkachenko, Jonathan M. Ward, and Síle Nic Chormaic ${ }^{\dagger}$ \\ Light-Matter Interactions Unit, Okinawa Institute of Science and Technology Graduate University, Onna, \\ Okinawa 904-0495, Japan
}

(Received 14 December 2018; revised manuscript received 10 April 2019; published 18 June 2019)

\begin{abstract}
Optical nanofiber waveguides are widely used for near-field delivery and measurement of light. Despite their versatility and efficiency, nanofibers have a critical drawback - their inability to maintain light's polarization state on propagation. Here, we design a directional coupler consisting of two crossed nanofibers to probe the polarization state at the waist region. Directionality of coupling occurs due to asymmetric dipolar emission or spin locking when the evanescent field pattern breaks the mirror symmetry of the crossed-nanofiber system. We demonstrate that, by monitoring the outputs from the directional coupler, two nonorthogonal polarization states can be prepared at the nanofiber waist with a fidelity higher than $99 \%$. Based on these states, we devise a simple and reliable method for complete control of the polarization along a nanofiber waveguide.
\end{abstract}

DOI: 10.1103/PhysRevApplied.11.064041

\section{INTRODUCTION}

Tapered optical fibers are unique because they allow for the smooth transition of light from macroscale to microscale or nanoscale systems. Owing to the strong evanescent field around the ultrathin waist region, such fibers are efficient and versatile tools for optical manipulation [1-3], sensing [4,5], nonlinear optics [6,7], microcavities [8], atomic physics [9-13], and various studies of light-matter interactions in both classical and quantum regimes [14-16]. As light propagates from the fiber pigtail to the waist region [Fig. 1(a)], the mode volume reduces, and the intensity of the evanescent field increases [17]. Optical power can be transferred to the waist without significant losses, even for ultrathin fibers. However, the other important parameter of the field - its polarization - is usually impossible to control. The polarization of the evanescent field is crucial for many nanofiber-based studies including microcavity mode excitation [18] and interactions with nanoparticles [19] or atomic ensembles $[20,21]$.

As simple as it may seem, the problem of polarization uncertainty in tapered fibers has challenged the scientific community for decades. To solve it, one must establish

\footnotetext{
*georgiy.tkachenko@oist.jp

${ }^{\dagger}$ Also at Institut Néel, Université Grenoble Alpes, F-38042 Grenoble, France.

Published by the American Physical Society under the terms of the Creative Commons Attribution 4.0 International license. Further distribution of this work must maintain attribution to the author(s) and the published article's title, journal citation, and DOI.
}

a direct link between the near field at the ultrathin waist and the far field where macroscopic detectors and controllers are typically placed. In this work, we explore directional coupling between two crossed nanofibers, and, consequently, develop a reliable method for achieving an arbitrary polarization state in the evanescent field of a single-mode nanofiber. The physics behind this directionality features the interplay of two phenomena: the well-known spin-momentum locking, and the newly found mirror-symmetry breaking of a light-matter system by an induced electric dipole.

We begin by introducing optical nanofibers and their fabrication in Sec. II, and discuss how the polarization of light propagates in adiabatically tapered fibers. We emphasize that the polarization transformation in such fibers, as in any optical elements free of depolarization and dichroism, is equivalent to rotations of the Poincare sphere. In Sec. III, we devise a simple, two-step procedure, which allows for reversing the above rotational transformations via consecutive mapping of two nonorthogonal polarization states. By compensating an arbitrary transformation, one achieves complete control over the polarization state. Section IV is dedicated to the directional coupling between two single-mode optical nanofibers crossed at right angles. We present a detailed experimental and numerical study of the directional coupler's operation. The results indicate how to securely identify a pair of nonorthogonal states required for the two-step compensation procedure to be applicable in the case of a nanofiber waveguide. Section $\mathrm{V}$ presents a practical demonstration of the polarization control. We discuss its precision and accuracy, as well as the experimental evidence related to polarization evolution in a tapered optical fiber. 


\section{POLARIZATION OF LIGHT IN A NANOFIBER WAVEGUIDE}

Nanofibers are generally produced from conventional optical fibers by controlled heating and pulling [22]. A typical, single-mode nanofiber consists of a cylindrical submicron-diameter waist connected to fiber pigtails by two taper regions, see Fig. 1(a), where the Cartesian coordinate system $(x, y, z)$ originates in the middle of the waist and $z$ is parallel to the fiber axis. For this work, the tapered fibers are prepared from a step-index cylindrical optical fiber with a cutoff wavelength of $920 \pm 50 \mathrm{~nm}$. The cylindrical waist regions are about $2 \mathrm{~mm}$ long with radii of $159 \pm 3 \mathrm{~nm}$, as measured by a scanning electron microscope. The input pigtail is coupled to a collimated Gaussian beam from a continuous-wave laser (980-nm wavelength). Each fiber is kept as short, straight, and strain-free as possible. Such precautions are common practice in nanofiber experiments where polarization (or mode) transformations are undesirable.

The key questions to be addressed are (i) how does the polarization of guided light change upon propagation through a tapered fiber? and - more importantly-(ii) how can this change be controlled? A polarization state can be treated as a unit vector $\mathbf{s}=\left(1, S_{1}, S_{2}, S_{3}\right)$, where $S_{1,2,3}$ are the Stokes parameters, which define a point on the Poincare sphere, $\mathcal{P}$, see Fig. 1(b). The azimuthal $(2 \psi)$ and polar $(2 \chi)$ angles on the sphere are directly linked to the orientation, ellipticity, and handedness (through the sign of $\chi$ ) of the polarization ellipse
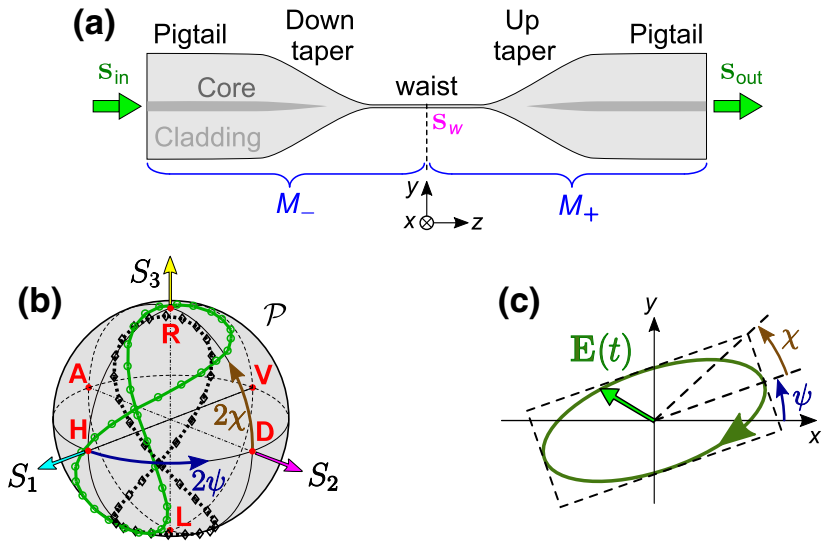

FIG. 1. (a) A tapered optical fiber with an ultrathin waist does not, generally, maintain the polarization of guided light. Transformation of the input polarization state, $\mathbf{s}_{\text {in }}$, to that at the waist, $\mathbf{s}_{w}$, is described by the Mueller matrix $M_{-}$. Another matrix, $M_{+} \neq M_{-}$, describes the transformation into the output state, $\mathbf{s}_{\text {out }}$. (b) The trajectories traced on the Poincaré sphere, $\mathcal{P}$, by the input ( $\mathbf{s}_{\text {in }}$, diamonds and dotted line) and the output ( $\mathbf{s}_{\text {out }}$, circles and solid line) polarization states are different, even for short and straight nanofibers. (c) Polarization ellipse defined by the angles $\psi$ and $\chi$, which correspond to half the azimuthal and polar angles on $\mathcal{P}$. traced in the $(x, y)$ plane by the tip of the electric field vector, $\mathbf{E}(t)$, see Fig. 1(c), in the following way: $\mathbf{s}=$ $(1, \cos 2 \psi \cos 2 \chi, \sin 2 \psi \cos 2 \chi, \sin 2 \chi)$.

First, we compared the polarization state at the input, $\mathbf{s}_{\text {in }}$, and the output, $\mathbf{s}_{\text {out }}$, of our tapered fibers. In order to probe a large part of $\mathcal{P}, \mathbf{s}_{\text {in }}$ is driven around the Poincaré sphere in a figure-of-eight trajectory by passing the horizontally polarized $(\mathbf{H})$ input beam through a rotating quarter-wave plate (QWP). The trajectory is recorded by means of a commercial polarization analyzer. For every fiber under test, the output trajectory repeated the shape of the input, but always exhibited a significant shift on the sphere [see a typical example in Fig. 1(b)], regardless of our efforts to maintain the polarization using the fore-mentioned precautions.

To understand how the shape of the trajectory on $\mathcal{P}$ is maintained, let us note that the fibers are designed according to the adiabatic condition, which implies energy transfer with minimum loss. Adiabaticity is ensured by keeping the taper angle below the critical value $[23,24]$ and the transmission at $980-\mathrm{nm}$ wavelength above $97 \%$ throughout the pulling process. Both pigtails and the cylindrical waist region are single mode, hence losses can only occur in the tapers via coupling to radiation modes and higher-order modes, which cannot propagate along the single-mode fiber. The guided electric field, $\mathbf{E}(z)$, in adiabatic single-mode fibers can be described as a combination of two orthogonal, quasilinearly polarized fundamental eigenmodes, $\mathbf{H E}_{11}^{x}$ and $\mathbf{H E}_{11}^{y}$ [17]:

$$
\mathbf{E}(z)=\alpha(z) \mathbf{H E}_{11}^{x}+\beta(z) \mathbf{H E}_{11}^{y},
$$

where $\alpha$ and $\beta$ are variable complex amplitudes. The polarization ellipse is associated with the Jones vector

$$
\mathbf{j}=\frac{1}{\sqrt{|\alpha|^{2}+|\beta|^{2}}}\left[\begin{array}{l}
\alpha \\
\beta
\end{array}\right] .
$$

Its evolution upon propagation from $z_{0}$ to $z$ through the fiber can be written as

$$
\mathbf{j}(z)=u_{\text {fiber }} \mathbf{j}\left(z_{0}\right),
$$

and, given that adiabatic fibers have transmission close to unity, $u_{\text {fiber }}$ must be a $2 \times 2$ unitary matrix:

$$
u_{\text {fiber }}^{\dagger} u_{\text {fiber }}=I,
$$

where $I$ is the identity matrix. Therefore, transformations of the Stokes vectors in adiabatically tapered fibers are restricted to the 3D rotation [SO(3)] group [25]. Rotations of the Poincaré sphere $\left(\mathcal{P} \rightarrow \mathcal{P}^{\prime}\right)$ preserve angles between the Stokes vectors, and, consequently, the shape of the trajectories for $\mathbf{s}_{\text {in }}$ and $\mathbf{s}_{\text {out }}$ in Fig. 1(b). This preservation of angles is the key condition for the polarization control that we achieve. 


\section{TWO-STEP POLARIZATION COMPENSATION}

The polarization state at the nanofiber waist, $\mathbf{s}_{w}$, can be linked to the input and output states in terms of Mueller calculus [26]: $\mathbf{s}_{w}=M_{-} \mathbf{s}_{\text {in }}$, and $\mathbf{s}_{\text {out }}=M_{+} \mathbf{s}_{w}$, where $M_{-}$ and $M_{+}$are $4 \times 4$ matrices describing the Stokes vector evolution before $(z<0)$ and after $(z>0)$ the waist. These two matrices do not correlate and cannot be determined if one only measures $\mathbf{s}_{\text {in }}$ and $\mathbf{s}_{\text {out }}$. Consequently, in order to control $\mathbf{s}_{w}$, one has to probe the evanescent field.

Theoretically, $M_{-}$can be found by measuring several sets of $\left(\mathbf{s}_{\mathrm{in}}, \mathbf{s}_{w}\right)$ [26]. However, this procedure is not always realistic. Instead of controlling the target polarization state by deducing $M_{-}$, in this work we follow a different strategy, which is more practical and, as will become clear, is the only option for nanofiber waveguides. Namely, we reverse the transformation of the Poincare sphere, $\mathcal{P} \rightarrow$ $\mathcal{P}^{\prime}$, thus achieving $\mathcal{P} \rightarrow \mathcal{P}$ and $\mathbf{s}_{w}=\mathbf{s}_{\text {in }}$.

From a geometrical point of view, the orientation of a sphere can be completely defined by two independent angles, i.e., latitude and longitude. It is, therefore, logical to realize the $\mathcal{P} \rightarrow \mathcal{P}$ mapping in two steps:

1. tilting of one axis by an angle $\varphi_{1}$ [Fig. 2(a)];

2. rolling of the two other axes about the first one by an angle $\varphi_{2}$ [Fig. 2(b)]. (a)

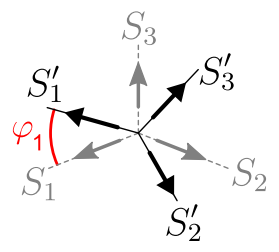

(b)

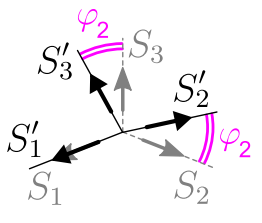

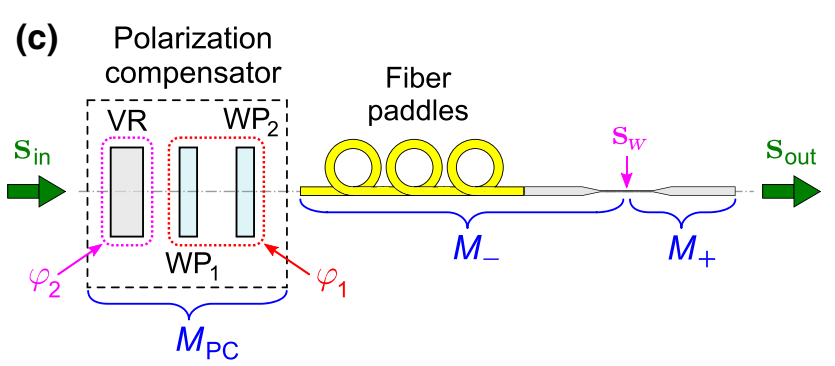

FIG. 2. (a),(b) A unitary transformation of the Poincare sphere $\mathcal{P} \rightarrow \mathcal{P}^{\prime}$ can be decomposed into two independent rotations by angles $\varphi_{1}$ and $\varphi_{2}$. To reverse the transformation, we adjust $\varphi_{1}$ in order to achieve one state (here $\mathbf{H}$ or $S_{1}=1$ ) maintained (that is $\mathbf{H} \rightarrow \mathbf{H}, S_{1}^{\prime}=S_{1}=1$ ). Then, while keeping $\varphi_{1}$ fixed, $\varphi_{2}$ is adjusted until $S_{2,3}^{\prime}=S_{2,3}$. (c) To control polarization at the nanofiber waist, we compensate for the unknown matrix $M_{-}$by sequentially picking $\varphi_{1}$ and $\varphi_{2}$ using two quarter-wave plates $\left(\mathrm{WP}_{1,2}\right)$ and a variable retarder (VR). Fiber paddles allow randomization of $M_{-}$when studying the precision of the control.
Importantly, this two-step procedure for reversing unknown polarization transformations is not restricted to nanofibers, but can be applied to any optical element free of depolarization and dichroism. In practice, we realize the procedure by means of a free-space polarization compensator (PC) consisting of a variable retarder (with the fast axis parallel to $x)$ and a pair of quarter-wave plates $\left(\mathrm{WP}_{1,2}\right)$, see Fig. 2(c). The compensator is characterized by a unitary Jones matrix, $u_{\mathrm{PC}}$, or a Mueller matrix, $M_{\mathrm{PC}}$. In step (1), $\mathrm{WP}_{1}$ and $\mathrm{WP}_{2}$ are independently rotated until the input horizontal polarization $\left(\mathbf{s}_{\text {in }}=\mathbf{H} ; S_{1}=1\right)$ is mapped onto itself at the nanofiber waist ( $\left.\mathbf{s}_{w}=\mathbf{H} ; S_{1}^{\prime}=1\right)$. Next, in step (2), an input state with $\left|S_{1}\right| \neq 1$ is selected, and the retardance of VR is adjusted. This drives $\mathbf{s}_{w}$ along the circle in the plane parallel to $\left(S_{2}, S_{3}\right)$ until eventually $S_{2,3}^{\prime}=S_{2,3}$, and thus $\mathbf{s}_{w}=\mathbf{s}_{\text {in }}$ due to $M_{\mathrm{PC}}=M_{-}^{-1}$. In fact, $\mathcal{P} \rightarrow \mathcal{P}$ mapping can be performed with any pair of nonorthogonal states, i.e., such states that do not lie on the same diameter of the Poincaré sphere, or - in mathematical terms - have a nonzero inner product (see the proof in the Appendix).

\section{CROSSED-NANOFIBER DIRECTIONAL COUPLER}

In practice, to identify two nonorthogonal polarization states at the waist of a nanofiber, we cross it with a near-identical nanofiber at right angles, as sketched in Fig. 3(a).Near-field probing one (input) ultrathin fiber with another (output) one is not new, see, for instance, its application for the purpose of profilometry [27,28]. In this work, for the first time, we consider symmetry of such a system (hence the importance of the right-angle crossing) and use output signals from both ends of the probe fiber. The efficiency of near-field coupling to guided modes, which produce these signals is very low, typically under $0.1 \%$, see Figs. 6(d) and 6(e). However, it is sufficient for unambiguous identification of nonorthogonal $\mathbf{H}$ and $\mathbf{R}_{M}$ (see below) polarization states at the waist of the input fiber.

The system of two crossed fibers has a symmetry plane, $(x, z)$, shown as the dash-dotted line in Figs. 3(b) and 3(c). When the input beam is $\mathbf{H}$ or $\mathbf{V}$ polarized, the overall symmetry of the light-matter system is preserved and the optical power values, $P_{+}$and $P_{-}$, measured at the ends of the output fiber, are equal: $P_{+}=P_{-}$. Otherwise, the symmetry is broken and in general $P_{+} \neq P_{-}$. Interestingly, this directional coupling of light can be separated into two independent effects associated with orientation $(\psi)$ and shape $(\chi)$ of the polarization ellipse. Let us now consider the two special cases of linear $(\psi \neq$ const; $\chi=0)$ and circular $(\chi= \pm \pi / 4)$ polarizations.

For a tilted linear polarization [Fig. 3(b)], the mirror symmetry is broken by the emission pattern of an electric dipole induced at the crossing point. As a result, two counter-propagating modes of nonequal power are generated in the output fiber. A wave with reflection angle, $\theta$, 
(a)

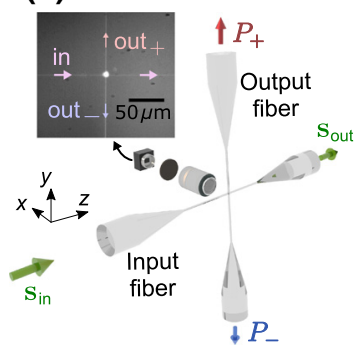

(b)

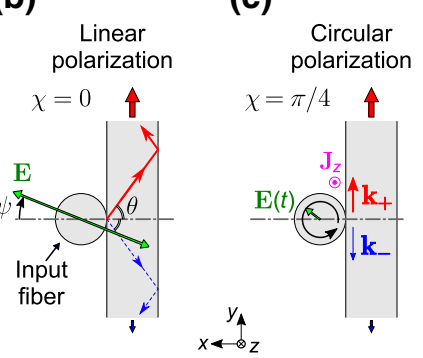

FIG. 3. (a) Crossed-nanofiber optical coupler and the imaging system consisting of a $\times 20$ objective lens, a linear polarizer, and a video camera. The camera image (top left) shows nanofibers and the scattering spot from the crossing. (b) Tilted linear polarization states of guided light break the mirror symmetry of the system, thus leading to power imbalance between the output channels. (c) Circular polarization breaks the symmetry too. Directional coupling occurs due to locking of the transverse spin angular momentum, $\mathbf{J}_{z}$, to the output wave vector, here $\mathbf{k}_{+}$.

carries energy proportional to $\sin ^{2}(\theta+\psi)$. Assuming that all angles allowed in this nanofiber equally contribute to the energy transfer, the net power radiated along the \pm direction is proportional to $\int_{\theta_{c}}^{\pi / 2} \sin ^{2}(\theta \pm \psi) d \theta$, where $\theta_{c}$ is the critical angle. Therefore, the output power sum,

$$
P_{\Sigma}=\left(P_{+}+P_{-}\right) \propto(\cos 2 \psi+\text { const }),
$$

which has a maximum (minimum) for $\mathbf{H}(\mathbf{V})$ polarization. This effect, which we dubbed "asymmetric dipolar emission" in order to emphasize its geometrical origin, provides an alternative way to achieve directionality without spin-momentum locking [29]. This possibility has been overlooked in earlier works on directional coupling in similar systems [30,31].

For circular polarization, the electric field vector, $\mathbf{E}(t)$, traces a circle about the longitudinal axis, $z$, see Fig. 3(c). In free space, such a field only contains the longitudinal component of the photon spin, $\mathbf{J}_{z}$. The evanescent field may also contain a significant transverse spin component, $\mathbf{J}_{\text {trans }}$, which appears due to interaction between the real, $\operatorname{Re}(\mathbf{k})$, and imaginary, $\operatorname{Im}(\mathbf{k})$, parts of the wave vector, $\mathbf{k}$, at the interface between two different media [32]. Interestingly, $\operatorname{Re}(\mathbf{k}), \operatorname{Im}(\mathbf{k})$, and $\mathbf{J}_{\text {trans }}$ must form a right-handed system [33]. As a result, the direction of $\mathbf{J}_{\text {trans }}$ defines the direction of the propagating wave [30,34]. This phenomenon is known as "spin locking" or the "quantum spin Hall effect" of light [35]. With regard to our crossed nanofibers, spin locking causes $P_{-} \neq P_{+}$, since the vector $\mathbf{J}_{z}$ is simultaneously the longitudinal spin for the input nanofiber and the transverse spin for one of the counterpropagating, $x$-polarized modes of the output nanofiber. Simple trigonometric considerations yield that the output power difference produced by the spin-locking effect,

$$
P_{\Delta}=\left(P_{+}-P_{-}\right) \propto \sin 2 \chi,
$$

with maximum and minimum values at $\chi=\pi / 4$ ( $\mathbf{R}$ polarization state) and $\chi=-\pi / 4$ (L state), respectively.

For elliptical polarization $(\psi, \chi \neq 0)$, both asymmetric dipolar emission and spin-locking effects may influence the output power balance. In order to confirm the above analytical predictions for the special cases and generalize the

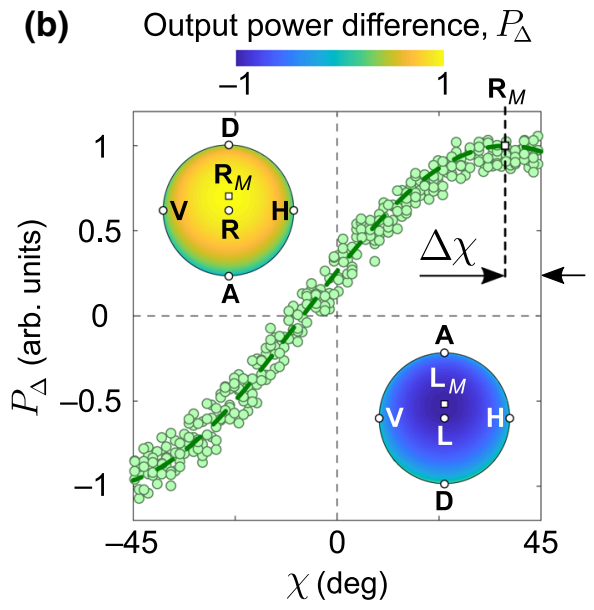

FIG. 4. Operation of the crossed-nanofiber coupler. (a) Measured (diamonds) and simulated (solid curve) values of the output power sum, $P_{\Sigma}$, versus orientation of the linear polarization, $\psi$. The numerical simulation over the Poincaré sphere (insets) shows that $P_{\Sigma}$ has the global maximum for the horizontal and the global minimum for the vertical polarization states. The intensity of scattering from the fiber crossing (dots - measured, dashed curve — simulated) is exactly opposite in phase with respect to $P_{\Sigma}$. (b) Measured (circles) and simulated (dashed curve) values of the output power difference, $P_{\Delta}$, versus the polarization ellipticity. The simulated global maximum corresponds to the state $\mathbf{R}_{M}$, which is shifted from $\mathbf{R}$ towards $\mathbf{D}$ due to the interplay between the two mechanisms of mirror symmetry breaking. 
(a)

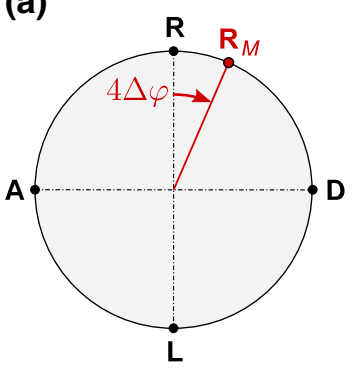

(b)

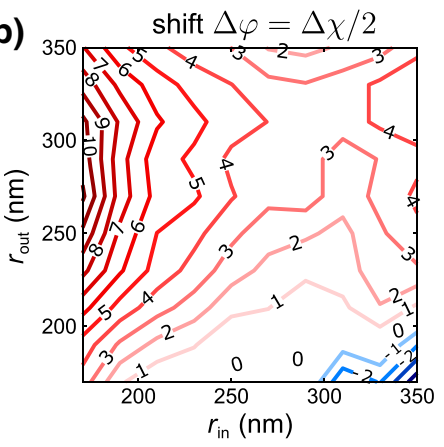

FIG. 5. Shift of the maximum $P_{\Delta}$ for $\mathbf{s}_{\text {in }}$ tracing the circle in the $S_{1}=0$ plane of the Poincare sphere. (a) On the sphere, the shift appears as $2 \Delta \chi=4 \Delta \varphi$, where $\Delta \varphi$ is the corresponding orientation of the HWP in the polarization generator. (b) Simulated map of $\Delta \varphi$ versus radii of the input $\left(r_{\text {in }}\right)$ and output $\left(r_{\text {out }}\right)$ nanofibers. Noteworthy, the shift can be negative, in which case $\mathbf{R}_{M}$ is located between $\mathbf{R}$ and $\mathbf{A}$. However, in practice, the coupling efficiency of the crossed fibers in this regime is too weak to be detected, see Figs. 6(d) and 6(e).

understanding of the directional coupler's operation, finiteelement numerical simulations of $P_{ \pm}$for $\mathbf{s}_{w}$ covering the whole Poincaré sphere are performed. The results for variable $\psi$ and $\chi$ are shown in Figs. 4(a) and 4(b) [36].The insets in Fig. 4(a) demonstrate that $\mathbf{H}$ and $\mathbf{V}$ correspond to the global extrema of $P_{\Sigma}$ and each of these two states can be identified and used in the first step of the polarization compensation. Experimentally, $\psi$ is varied while keeping $S_{3}=0$ by sending the $\mathbf{H}$-polarized input beam through a rotating half-wave plate (HWP). The data (diamonds) agree with the simulation (solid curve). Thus, $P_{\Sigma}$ can be readily used for $\mathbf{H} \rightarrow \mathbf{H}$ mapping, which can be further verified by monitoring the intensity of scattering from the fiber crossing, $I$, see the experimental (dots) and theoretical (dashed curve) results in Fig. 4(a). We measured $I$ as the total brightness of the camera image captured through a linear polarizer parallel to the $y$ axis [11].

The simulations of $P_{\Delta}$ [see insets in Fig. 4(b)] reveal that, due to the interplay between the asymmetric dipolar emission and the spin-locking effects, the global maximum (minimum) of $P_{\Delta}$ is shifted from the expected $\mathbf{R}(\mathbf{L})$ to $\mathbf{R}_{M}$ $\left(\mathbf{L}_{M}\right)$ by $\Delta \chi \approx 8^{\circ}$. This value depends on the radii of the nanofibers and is under $10^{\circ}$ for the region where significant coupling can be achieved [see Figs. 5(b), 6(d), and 6(e)]. Experimentally, $\chi$ is varied while keeping $S_{1}=0$ by rotation of a HWP in front of a QWP fixed at $45^{\circ}$ to the $x$ axis, as depicted in Fig. 8(g). In order to avoid systematic errors associated with $\Delta \chi$, in the second step of the polarization control, we mapped $\mathbf{R}_{M} \rightarrow \mathbf{R}_{M}$ instead of $\mathbf{R} \rightarrow \mathbf{R}$.

All data presented in Fig. 4 are collected while keeping the fiber crossing point fixed. Now let us check whether the choice of the crossing point makes a difference. For instance, if the polarization at the input fiber waist depends on the longitudinal position, $z$, the curves for $P_{\Sigma, \Delta}$ versus

the HWP orientation, $\varphi_{\mathrm{HWP}}$, will have variable phase shifts. Alterations to $P_{\Sigma, \Delta}$ will also appear if the directional coupling depends on the vertical position of the output fiber, or, effectively, its radius at the crossing. We test both possibilities using one period of the HRVL trajectory on the Poincaré sphere [Figs. 8(d) and 8(f)]. First, we displaced the output fiber along its axis, thus varying $\Delta y=\left(y-y_{0}\right)$ while keeping the longitudinal position of the crossing point fixed, so that $\Delta z=\left(z-z_{0}\right)=$ const, where $y_{0}, z_{0}$ are random initial coordinates. As shown in Fig. 6(a), the measured $P_{\Delta}$ is independent of $\Delta y$, up to an amplitude scaling factor. The same behavior is observed when the crossing point is displaced along $z$ with $\Delta y=$ const, see Fig. $6(\mathrm{~b})$. These results indicate that $\mathbf{s}_{w}$ is maintained throughout the coupling region, which we define as the $y \times z$ area where light can still couple to the output fiber. We find that the amplitudes of $P_{+}$and $P_{-}$are nonzero within $4 \times 4 \mathrm{~mm}^{2}$ area covering the waist and thinner parts of the tapers, see the radius profile in Fig. 6(c).

According to our numerical simulations presented in Fig. 6(d), the coupling efficiency, $\eta=P_{\Sigma} / P_{T}$ (where $P_{T}$ is the optical power transmitted through the input fiber) is maximum for an input nanofiber radius, $r_{\mathrm{in}}^{\max } \approx 210 \mathrm{~nm}$, and an output nanofiber radius, $r_{\text {out }}^{\max } \approx 220 \mathrm{~nm}$. We measure $\eta$ over the whole coupling range with a step of 0.25 $\mathrm{mm}$ in $y$ and $z$. The resulting efficiency map shown in Fig. 6(e) agrees reasonably with the simulations. Notably, the whole explored range for the nanofiber radii, $r$, corresponds to the single-mode regime, since the normalized frequency parameter, $V=(2 \pi r / \lambda) \sqrt{1.45^{2}-1}<2.356$, is below the cutoff value of 2.405 [37]. In fact, the polarization compensation is valid only for single-mode input nanofibers, with a minimum wavelength-to-diameter ratio of $\pi \mathrm{NA} / 2.405$ (about 1.372 for glass in air).

\section{POLARIZATION CONTROL}

Figure 7 illustrates the precision of the polarization control we achieve. In order to estimate the precision, the input pigtail is spliced to fiber paddles [Fig. 2(c)] that allows us to produce a random $M_{-}$. For each random setting of the paddles, the two-step compensation procedure is performed by $\mathbf{H} \rightarrow \mathbf{H}$ and $\mathbf{R}_{M} \rightarrow \mathbf{R}_{M}$ mapping. Then, $\mathbf{s}_{\text {out }}$ is measured for the three principal states: $\mathbf{H}, \mathbf{D}$, and $\mathbf{R}$. The resulting statistics over 26 sets [see Fig. 7(a)] gives the following deviations from the mean: $1.16 \pm 1.43^{\circ}$, $6.03 \pm 3.82^{\circ}$, and $4.29 \pm 2.37^{\circ}$ for $\mathbf{H}, \mathbf{D}$, and $\mathbf{R}$, respectively. The fidelities (defined as the cosine of the mean angular distances) for these states are $0.9998,0.9945$, and 0.9972 , respectively. The offsets from the target states (about $10^{\circ}$ for this fiber) are due to the unknown, constant matrix $M_{+}$.

Besides the principal states, the method is tested for a figure-of-eight trajectory [black diamonds and dotted line in Figs. 1(b) and 7(b)]. The randomly arranged paddles 


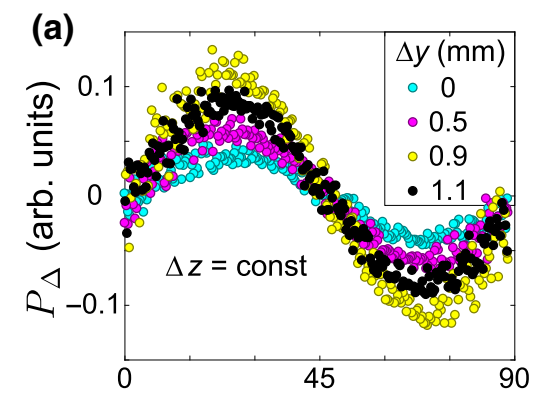

(c)
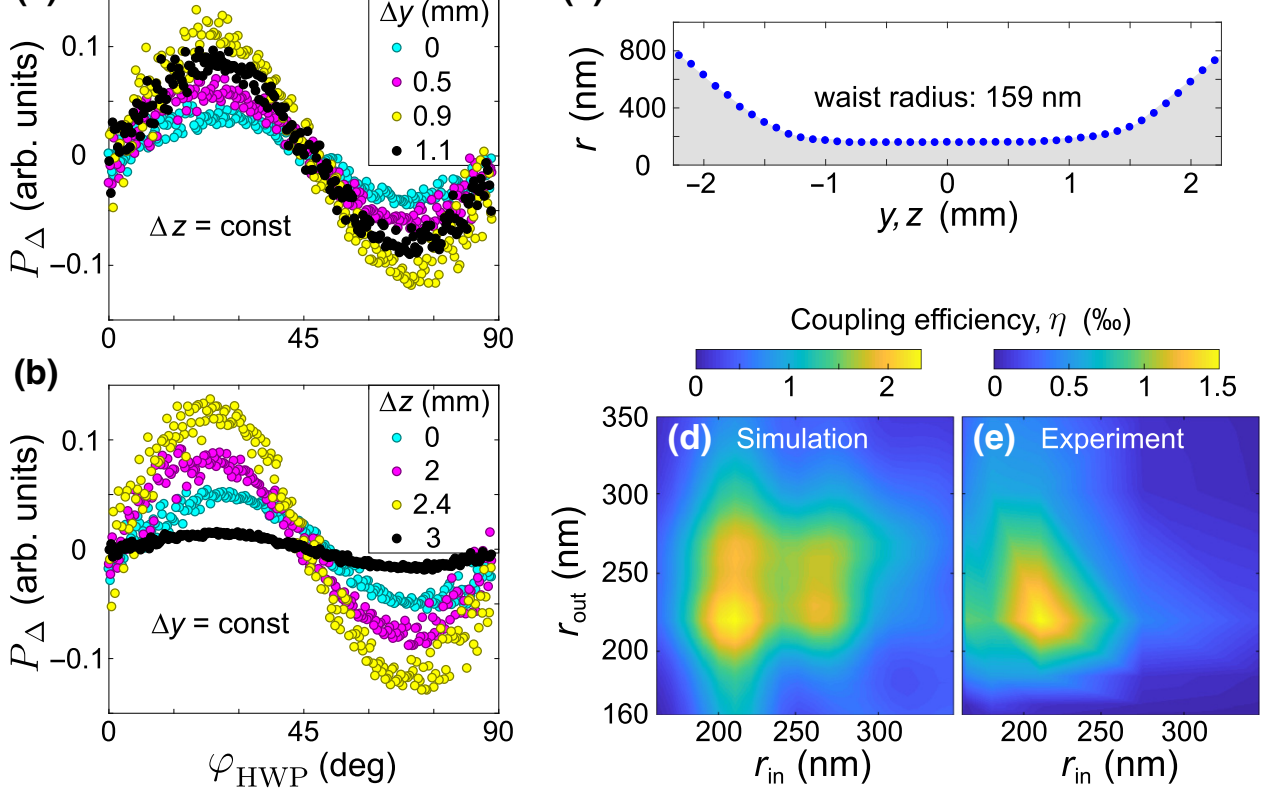

FIG. 6. The role of crossing-point position and fiber thickness. (a) Output power difference, $P_{\Delta}$, measured at a fixed point $(0,0, \Delta z)$ on the input fiber with the output fiber being displaced vertically by $\Delta y$. Although the amplitude of $P_{\Delta}$ depends on $\Delta y$, its shape and phase are preserved. Therefore, polarization information gathered by the output fiber does not depend on its radius. (b) The input fiber is probed at various $\Delta z$ by the output fiber of a fixed radius. The behavior of $P_{\Delta}$ indicates that the polarization state is maintained throughout the whole coupling region. (c) A typical nanofiber radius profile measured by a scanning electron microscope. (d),(e) Simulated and measured efficiency of the directional coupler, $\eta=P_{\Sigma} / P_{T}$, dependent on the radii of the nanofibers.

could move the uncompensated trajectory to any part of the sphere [see the blue solid curve in Fig. 7(b) as an example]. Compensation with $\mathbf{H} \rightarrow \mathbf{H}$ and $\mathbf{R}_{M} \rightarrow \mathbf{R}_{M}$ brings the trajectory (red filled squares) close to the initial one. This result indicates that the second (straight) part of the tapered fiber (corresponding to $M_{+}$) contributed only a minor transformation to $\mathbf{s}_{w}$. The case where the correction to $\Delta \chi$ is ignored is also checked. In this case, the initial state is $\mathbf{R}$, and $\mathbf{R} \rightarrow \mathbf{R}_{M}$ mapping is achieved by locating the maximum of $P_{\Delta}$. This mapping causes
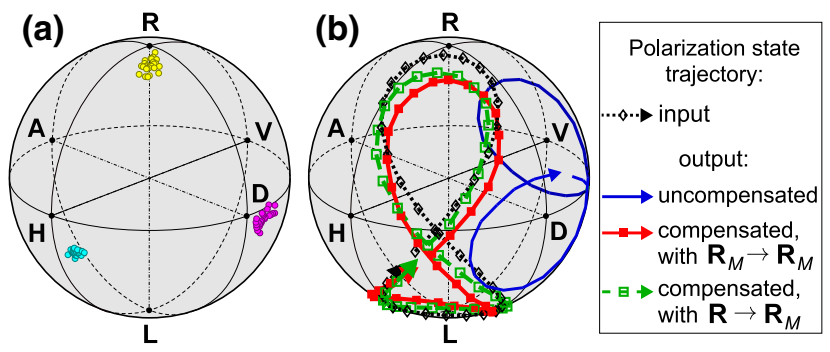

FIG. 7. Precision of the polarization control. (a) Statistics for the principal states measured at the end of the input fiber after compensation for a random $M_{-}$. (b) Recovering an arbitrary input trajectory (black diamonds) for $\mathbf{s}_{w}$ using compensation with $\mathbf{R}_{M} \rightarrow \mathbf{R}_{M}$ (solid red squares) or $\mathbf{R} \rightarrow \mathbf{R}_{M}$ (empty green squares) mapping. systematic errors for $\varphi_{2}$, see the compensated trajectories in Fig. 7(b).

In order to demonstrate the accuracy of the polarization control, we perform a detailed study summarized in Fig. 8.In this study, the input polarization state, $\mathbf{s}_{\text {in }}$, is driven along a circular trajectory in one of the principal planes of the Poincaré sphere: $S_{3}=0, S_{2}=0$, or $S_{1}=$ 0 . This is achieved by the free-space polarization generator depicted in the top panels [that is, (a), (d), and (g)] for each trajectory. The experimental data for the directional coupler's outputs and the scattering intensity are collected after the polarization compensation procedure. We note that spin-locking and asymmetric dipolar emission effects produce comparable modulations of the directionality characterized by $P_{\Delta}$, see Figs. 8(c) and 8(f). The higher noise in the "-" channel [especially noticeable in Fig. 8(b)] is repeatable over numerous experimental attempts and independent of the detectors. The noise levels in the two channels are closer to each other for thicker nanofibers (with radii over $250 \mathrm{~nm}$ ). Therefore, we attribute this effect to higher sensitivity of thinner nanofibers to bends due to vibrations or sagging, and, perhaps, to unknown deviations of the generated trajectory from the target one.

Our results contradict the general belief that short lengths of straight tapered fibers do not change the polarization by much and, even if they do, the shifts of 

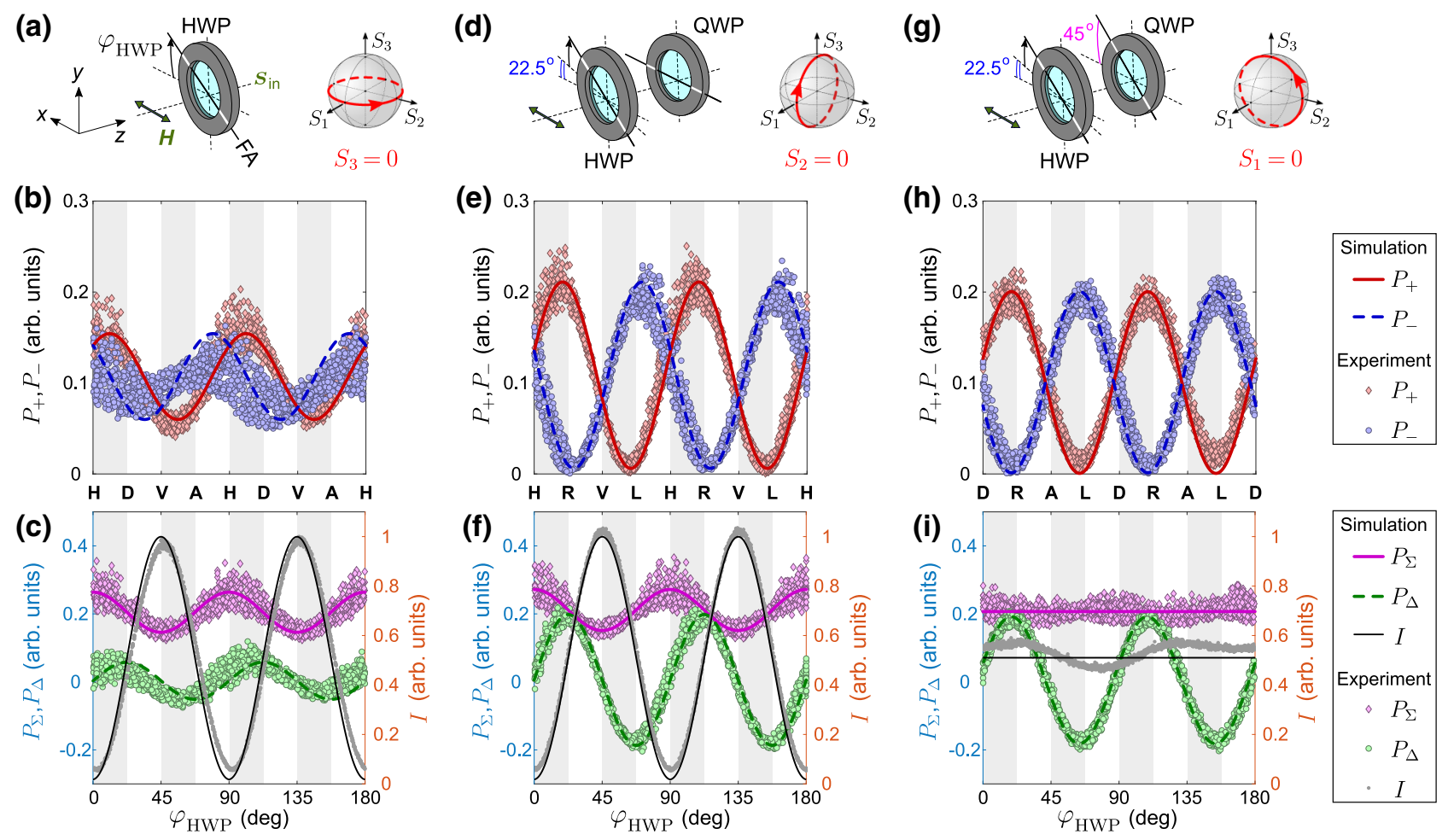

FIG. 8. Testing the crossed-nanofiber directional coupler after the polarization compensation. (a),(d),(g) The input polarization state, $\mathbf{s}_{\text {in }}$, traces a circle in one of the principal planes of the Poincare sphere, driven by the polarization generator represented by a rotating HWP and a static QWP. (b) Simulated (solid and dashed lines) and measured (diamonds and circles) optical powers at the "+" and "_" ends of the output fiber for $\mathbf{s}_{\text {in }}$ lying in the $S_{3}=0$ plane. (c) Simulated (lines) and measured (diamonds and circles) sum and difference of the output powers, along with the scattering intensity (dots). (e),(f) Same as (b),(c), but for the $S_{2}=0$ plane. The simulated $P_{\Delta}$ is maximum for the $\mathbf{R}$ state, and minimum for the $\mathbf{L}$ state. (h),(i) Same for the $S_{1}=0$ plane. The simulated maximum of $P_{\Delta}$ is slightly shifted from $\mathbf{R}$ towards $\mathbf{D}$ (by $\Delta \varphi$, see Fig. 5) due to the interplay between the asymmetric dipolar emission and the spin-locking effects. Here the experimental $P_{-,+, \Sigma, \Delta}$ are in units of voltage from photodetectors; simulated $P_{-,+}$are normalized by the experimental maxima; scattering intensity $I$ is normalized by its maximum. The gray bands are added as a guide to the eye.

the input state, $\mathbf{s}_{\text {in }}$, by the down and up tapers are equal, so that the state at the waist, $\mathbf{s}_{w}$, lies in the center between $\mathbf{s}_{\text {in }}$ and $\mathbf{s}_{\text {out }}$. According to our findings, presented in Figs. 1(b) and 7(b), the transformation matrices $M_{-}$and $M_{+}$lead to oppositely directed, nonequal shifts of significant magnitudes. Therefore, it is clear that experiments with high levels of precision (like those involving quantum emitters) do require near-field polarization control, which can now be achieved using the reported method.

\section{CONCLUSION}

We demonstrate a method for the complete control of polarization of light in a single-mode optical nanofiber waveguide, using directional coupling between two such nanofibers. Although based on complex physical phenomena (the spin-momentum locking and the newly discovered asymmetric dipolar emission), our method is surprisingly simple and highly reliable. We believe that it will have significant impact on the vast range of experimental systems based on optical nanofibers and evanescently coupled elements, in general. The demonstrated directional coupler itself is a promising platform for developing integrated photonic circuits.

\section{ACKNOWLEDGMENTS}

The authors thank S.P. Mekhail for help with automation of the experiment, K. Karlsson for assistance with nanofiber fabrication, and F. Le Kien for helpful discussions. This study is partially supported by the Okinawa Institute of Science and Technology Graduate University. G.T. is supported by the Japan Society for the Promotion of Science (JSPS) as an International Research Fellow (Standard, ID No. P18367).

F. Lei and G. Tkachenko contributed equally to this work.

\section{APPENDIX: POLARIZATION COMPENSATION REQUIRES NONORTHOGONAL STATES}

Our polarization compensation is based on sequential mapping of two input polarization states, $\mathbf{A}$ and $\mathbf{B}$, onto 
themselves at the waist of an optical nanofiber. As a result,

$$
\begin{aligned}
u \mathbf{A} & =\mathrm{e}^{i \delta_{A}} \mathbf{A}, \\
u \mathbf{B} & =\mathrm{e}^{i \delta_{B}} \mathbf{B},
\end{aligned}
$$

where the unknown phase factors, $\delta_{A}$ and $\delta_{B}$, do not change the state, and the Jones matrix, $u=u_{\text {fiber }} u_{\mathrm{PC}}$, describes the polarization transformation in the compensator $\left(u_{\mathrm{PC}}\right)$ and the tapered fiber until the waist $\left(u_{\text {fiber }}\right)$. Since $u$ is unitary, the inner product of these states

$$
\left\langle\mathbf{A}\left|u^{\dagger} u\right| \mathbf{B}\right\rangle=\langle\mathbf{A} \mid \mathbf{B}\rangle=\mathrm{e}^{i\left(\delta_{A}-\delta_{B}\right)}\langle\mathbf{A} \mid \mathbf{B}\rangle .
$$

If these two states are not orthogonal, that is

$$
\langle\mathbf{A} \mid \mathbf{B}\rangle \neq 0,
$$

then Eq. (A2) requires $\delta_{A}=\delta_{B}=\delta$. Under this condition, an arbitrary input state, $\mathbf{C}$, can be defined in the basis (A, B):

$$
\begin{aligned}
\mathbf{C} & =a \mathbf{A}+b \mathbf{B}, \\
u \mathbf{C} & =\mathrm{e}^{i \delta} \mathbf{C},
\end{aligned}
$$

where $a$ and $b$ are complex coefficients. Such a state $\mathbf{C}$ will be maintained in the polarization-compensated nanofiber.

[1] G. Brambilla, G. Senthil Murugan, J. S. Wilkinson, and D. J. Richardson, Optical manipulation of microspheres along a subwavelength optical wire, Opt. Lett. 32, 3041 (2007).

[2] A. Maimaiti, V. G. Truong, M. Sergides, I. Gusachenko, and S. Nic Chormaic, Higher order microfibre modes for dielectric particle trapping and propulsion, Sci. Rep. 5, 9077 (2015).

[3] Y. Ren, R. Zhang, C. Ti, and Y. Liu, Tapered optical fiber loops and helices for integrated photonic device characterization and microfluidic roller coasters, Optica 3, 1205 (2016).

[4] T. Yoshie, L. Tang, and S.-Y. Su, Optical microcavity: Sensing down to single molecules and atoms, Sensors 11, 1972 (2011).

[5] X.-C. Yu, B.-B. Li, P. Wang, L. Tong, X.-F. Jiang, Y. Li, Q. Gong, and Y.-F. Xiao, Single nanoparticle detection and sizing using a nanofiber pair in an aqueous environment, Adv. Mater. 26, 7462 (2014).

[6] J.-C. Beugnot, S. Lebrun, G. Pauliat, H. Maillotte, V. Laude, and T. Sylvestre, Brillouin light scattering from surface acoustic waves in a subwavelength-diameter optical fibre, Nat. Commun. 5, 5242 (2014).

[7] H. Zoubi and K. Hammerer, Quantum Nonlinear Optics in Optomechanical Nanoscale Waveguides, Phys. Rev. Lett. 119, 123602 (2017).

[8] M. Cai, O. Painter, and K. J. Vahala, Observation of Critical Coupling in a Fiber Taper to a Silica-Microsphere
Whispering-Gallery Mode System, Phys. Rev. Lett. 85, 74 (2000).

[9] F. Le Kien, V. Balykin, and K. Hakuta, Atom trap and waveguide using a two-color evanescent light field around a subwavelength-diameter optical fiber, Phys. Rev. A 70, 063403 (2004).

[10] S. M. Hendrickson, M. M. Lai, T. B. Pittman, and J. D. Franson, Observation of Two-Photon Absorption at Low Power Levels Using Tapered Optical Fibers in Rubidium Vapor, Phys. Rev. Lett. 105, 173602 (2010).

[11] E. Vetsch, S. T. Dawkins, R. Mitsch, D. Reitz, P. Schneeweiss, and A. Rauschenbeutel, Nanofiber-based optical trapping of cold neutral atoms, IEEE J. Sel. Top. Quant. Electron. 18, 1763 (2012).

[12] R. Kumar, V. Gokhroo, and S. Nic Chormaic, Multi-level cascaded electromagnetically induced transparency in cold atoms using an optical nanofibre interface, New J. Phys. 17, 123012 (2015).

[13] D. F. Kornovan, M. I. Petrov, and I. V. Iorsh, Transport and collective radiance in a basic quantum chiral optical model, Phys. Rev. B 96, 115162 (2017).

[14] X. Guo, M. Qiu, J. Bao, B. J. Wiley, Q. Yang, X. Zhang, Y. $\mathrm{Ma}, \mathrm{H}$. Yu, and L. Tong, Direct coupling of plasmonic and photonic nanowires for hybrid nanophotonic components and circuits, Nano Lett. 9, 4515 (2009).

[15] L. Tong, F. Zi, X. Guo, and J. Lou, Optical microfibers and nanofibers: A tutorial, Opt. Commun. 285, 4641 (2012).

[16] P. Solano, J. A. Grover, J. E. Hoffman, S. Ravets, F. K. Fatemi, L. A. Orozco, and S. L. Rolston, Advances in Atomic, Molecular, and Optical Physics (Elsevier, Cambridge, Massachusetts, US, 2017), p. 439.

[17] F. Le Kien, J. Q. Liang, K. Hakuta, and V. I. Balykin, Field intensity distributions and polarization orientations in a vacuum-clad subwavelength-diameter optical fiber, Opt. Commun. 242, 445 (2004).

[18] J. C. Knight, G. Cheung, F. Jacques, and T. A. Birks, Phasematched excitation of whispering-gallery-mode resonances by a fiber taper, Opt. Lett. 22, 1129 (1997).

[19] S. Wang, X. Pan, and L. Tong, Modeling of nanoparticleinduced Rayleigh-Gans scattering for nanofiber optical sensing, Opt. Commun. 276, 293 (2007).

[20] T. Nieddu, V. Gokhroo, and S. Nic Chormaic, Optical nanofibres and neutral atoms, J. Opt. 18, 053001 (2016).

[21] M. Sadgrove, S. Wimberger, and S. Nic Chormaic, Quantum coherent tractor beam effect for atoms trapped near a nanowaveguide, Sci. Rep. 6, 28905 (2016).

[22] J. M. Ward, A. Maimaiti, V. H. Le, and S. Nic Chormaic, Contributed review: Optical micro- and nanofiber pulling rig, Rev. Sci. Instrum. 85, 111501 (2014).

[23] J. D. Love, W. M. Henry, W. J. Stewart, R. J. Black, S. Lacroix, and F. Gonthier, Tapered single-mode fibres and devices, part 1: Adiabaticity criteria, IEE Proc. J Optoelectron. 138, 343 (1991).

[24] Y. Jung, G. Brambilla, and D. J. Richardson, Broadband single-mode operation of standard optical fibers by using a sub-wavelength optical wire filter, Opt. Express 16, 14661 (2008).

[25] J. J. Sakurai, Quantum Mechanics (Addison-Wesley, Reading, 1994). 
[26] R. A. Chipman, Handbook of Optics: Ch. 22 Polarimetry (McGraw-Hill, New York, New York, US, 2010).

[27] L. S. Madsen, C. Baker, H. Rubinsztein-Dunlop, and W. P. Bowen, Nondestructive profilometry of optical nanofibers, Nano Lett. 16, 7333 (2016).

[28] F. K. Fatemi, J. E. Hoffman, P. Solano, E. F. Fenton, G. Beadie, S. L. Rolston, and L. A. Orozco, Modal interference in optical nanofibers for sub-angstrom radius sensitivity, Optica 4, 157 (2017).

[29] M. F. Picardi, A. V. Zayats, and F. J. Rodríguez-Fortuño, Janus and Huygens Dipoles: Near-Field Directionality Beyond Spin-Momentum Locking, Phys. Rev. Lett. 120, 117402 (2018).

[30] J. Petersen, J. Volz, and A. Rauschenbeutel, Chiral nanophotonic waveguide interface based on spin-orbit interaction of light, Science 346, 67 (2014).

[31] M. Sadgrove, M. Sugawara, Y. Mitsumori, and K. Edamatsu, Polarization response and scaling law of chirality for a nanofibre optical interface, Sci. Rep. 7, 1 (2017).

[32] K. Y. Bliokh, F. J. Rodríguez-Fortuño, F. Nori, and A. V. Zayats, Spin-orbit interactions of light, Nat. Photonics 9 , 796 (2015).

[33] T. Van Mechelen and J. Zubin, Universal spin-momentum locking of evanescent waves, Optica 3, 118 (2016).

[34] D. O’Connor, P. Ginzburg, F. J. Rodríguez-Fortuño, G. A. Wurtz, and A. V. Zayats, Spin-orbit coupling in surface plasmon scattering by nanostructures, Nat. Commun. 5, 5327 (2014).

[35] K. Y. Bliokh, D. Smirnova, and F. Nori, Quantum spin hall effect of light, Science 348, 1448 (2015).

[36] For the ease of presentation, this figure shows experimental data collected after the polarization compensation.

[37] A. W. Snyder and J. D. Love, Optical Waveguide Theory (Chapman and Hall, London, UK, 1983). 